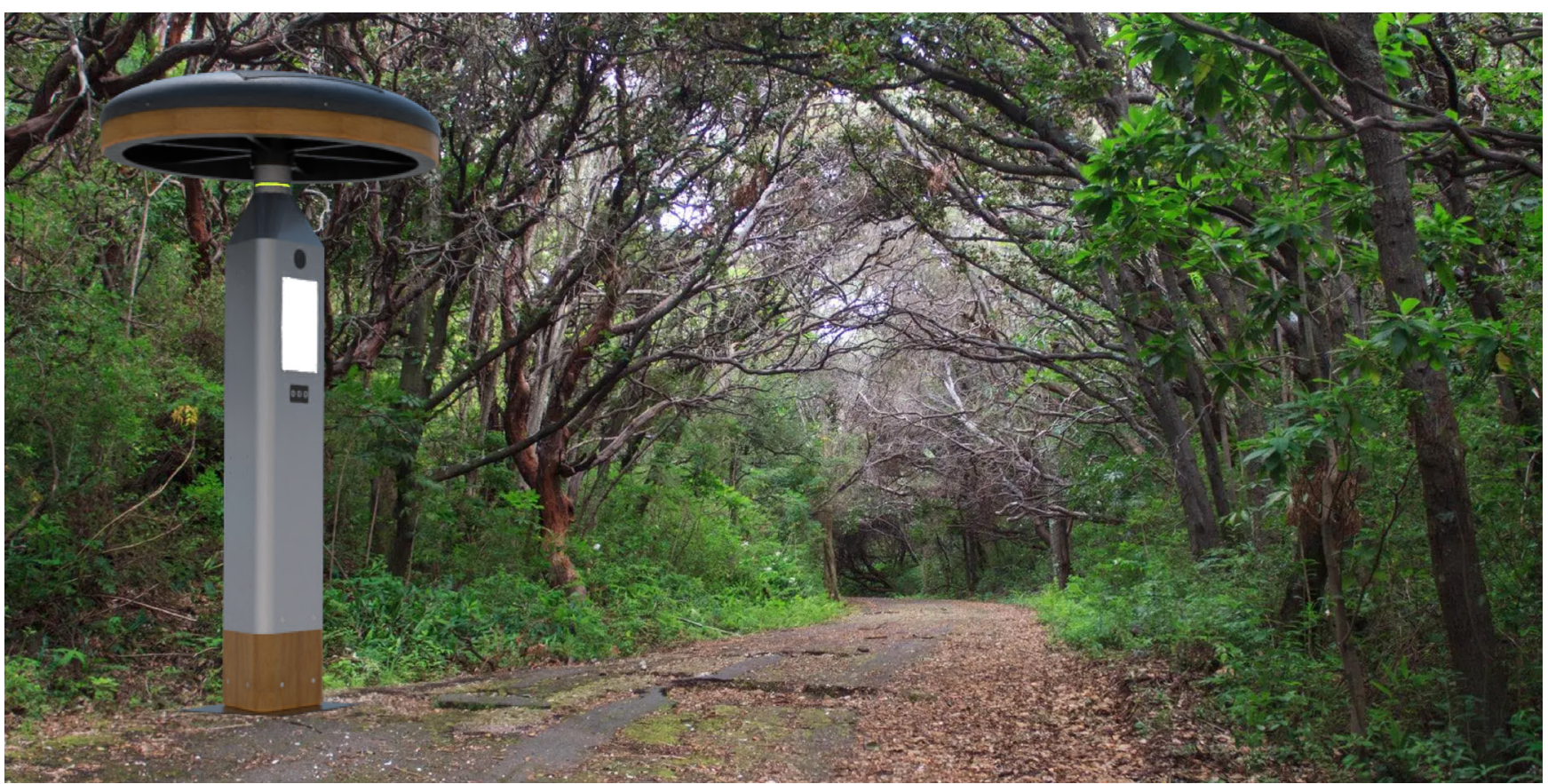

\title{
Diseño de un sistema inteligente para asistir, en situaciones de emergencia, a turistas que realizan senderismo en Costa Rica
}

Design of a smart system to assist tourists that perform trekking in Costa Rica on emergency situations

Braulio J. Quirós-Cordero ${ }^{1}$, María A. Piedra-Calderón ${ }^{2}$, Georgette A. Rojas-Muñoz ${ }^{3}$

B. Quirós-Cordero, M. Piedra-Calderón, G. Rojas-Muñoz “Diseño de un sistema inteligente para asistir, en situaciones de emergencia, a turistas que realizan senderismo en Costa Rica", IDI+, vol. 2, no 2, Ene., pp. 20-32, 2022.

doi) https: //doi.org/ 10.18845/ridip.v4i2.6072 Fecha de recepción: 16 de junio de 2021 Fecha de aprobación: 4 de noviembre de 2021

1. Braulio J. Quirós-Cordero

Estudiante de Ingeniería en Diseño Industrial Instituto Tecnológico de Costa Rica Cartago, Costa Rica braulio.j.qc@gmail.com (D) 0000-0002-0675-0529
2. María A. Piedra-Calderón

Estudiante de Ingeniería en Diseño Industrial Instituto Tecnológico de Costa Rica Cartago, Costa Rica andpica2@gmail.com (D) 0000-0001-9590-259x
3. Georgette A. Rojas-Muñoz Estudiante de Ingeniería en Diseño Industrial Instituto Tecnológico de Costa Rica Cartago, Costa Rica ariadneromu97@gmail.com (D) 0000-0002-5383-9438 


\section{Resumen}

El turismo en Costa Rica es una fuente de ingreso económico popular entre turistas tanto nacionales como extranjeros y, gracias a la diversidad de ecosistemas en el país, el senderismo es una de las principales actividades turísticas. El tipo de terreno montañoso en Costa Rica presenta diversidad de dificultades para que las personas puedan ubicarse. Sumado a este factor, la inexperiencia y falta de información sobre los lugares influyen en que las personas puedan perderse dentro de los senderos y encontrarse en situaciones de riesgo.

Debido a lo anterior, se encuentra una clara necesidad de brindar ayuda a las personas que frecuentan los senderos, para minimizar el riesgo de perderse dentro de estos, así como dar información relevante del lugar sobre instalaciones y rutas. Por lo cual, se planteó un objeto inteligente que cubre las necesidades definidas y mejora la experiencia de los turistas en las zonas montañosas. Mejorar los aspectos planteados trae beneficios en factores como seguridad integral, beneficio económico al minimizar los gastos en personal y equipo de rescate, además de mejorar la percepción del turismo de montaña.

Para abordar la problemática, se utilizó la metodología de design thinking, centrándose en pasos como conceptualizar la idea, definir la funcionalidad, la forma, detallado de la manufactura y simulación de diseño. Entre los principales aportes del proyecto están: brindar a los usuarios una mayor independencia durante sus recorridos en senderos, poder sentirse seguros en caso de alguna eventualidad y dar una respuesta rápida de las unidades de emergencia.

\section{Palabras clave}

Objeto inteligente; turismo; senderismo; zonas montañosas; diseño.

\section{Abstract}

Tourism in Costa Rica is a big economical source of income and with great popularity among tourists, both residents and non residents, and thanks to the diversity of ecosystems in the country, trekking is one of the main touristic activities performed. The mountainous land has several difficulties for people to get oriented. Added to this factor, the inexperience and lack of proper information of the place can lead people into getting lost inside the trails and finding themselves at risk.

There is a clear necessity to aid people that frequent the trails, to minimize the risk of losing inside those, as well as to give relevant information of the place such as installations and routes. A smart object is proposed to cover the defined needs and improve the experience of the tourists in mountainous areas. Improving these aspects can bring great benefits in aspects such as the integral safety, economical benefit by minimizing the expenses related to rescue teams and equipment, and enhancing the people's perception of mountain tourism. 
To approach the investigation, the design thinking method was used, focusing on steps like: conceptualizing the idea, defining the functionality, defining the shape, and design simulation. Some main contributions from the project are: giving users more independence during the trails, giving a feeling of safety in case of an eventuality, and a quick response from emergency units.

\section{Key words}

Smart object; tourism; trekking; mountainous areas; design.

\section{Introducción}

A lo largo de los años, Costa Rica se ha caracterizado por poseer una gran riqueza natural, lo que lo ha convertido en un atractivo turístico. El país se ha preocupado por tomar estos factores para su beneficio y poder no solo conservar la fauna, sino generar turismo centrado en las riquezas presentes. Según el Informe Anual de Estadísticas del SEMEC del 2017, el país cuenta con 127 áreas silvestres protegidas, de las cuales 41 reciben visitación turística [1].

Hoy en día, el turismo en el país es uno de los mayores pilares que aportan a la economía general y, a su vez, genera un crecimiento social. Entre los principales lugares que reciben visitación turística para actividades de senderismo se encuentran los parques nacionales, refugios de vida silvestre, reservas biológicas y reservas forestales. Según un censo realizado por el ICT al SINAC, en el 2017, se recibió un total de 999203 visitantes residentes y 1060 199 visitantes no residentes. Dentro de las áreas silvestres más visitadas, se encuentran el Parque Nacional Volcán Arenal, Parque Nacional Braulio Carrillo y el Parque Nacional Chirripó [2].

Parte de la meta del turismo en el país es dar una buena experiencia en los lugares, de manera que influya en la decisión de participar o no en alguna actividad turística. Uno de los factores que aporta a la buena experiencia es dar sensación de seguridad. Por lo que la seguridad turística debe cumplir con una serie de condiciones que brinden satisfacción al turista y que protejan su vida, salud, integridad física, psicológica y económica [3].

Debido a la situación actual de la pandemia por el COVID-19, el turismo en el país se ha visto afectado y sometido a diversidad de restricciones. Cuando se retomaron las actividades turísticas en Costa Rica, en este caso, en zonas montañosas, la población se vio atraída a realizar actividades de senderismo a estas zonas como un escape a las restricciones impuestas y la necesidad de permanecer en los hogares.

Aunque se pensara que no iban a ser muchos los rescates por realizar en las montañas, debido al poco flujo de personas en sectores montañosos, según La Nación, ha habido un incremento de los rescates de montaña desde el inicio de la pandemia, donde se dio un registro de 54 
casos entre marzo del 2020 y agosto del 2020 [4].

Entre algunos factores que influencian en el extravío de personas en zonas boscosas, La Nación menciona la falta de identificación de los senderos debido al rápido crecimiento de la maleza; seguir caminando sin preocuparse por retomar el camino original y factores climáticos como deslizamientos, lluvias y neblina que generan desorientación en zonas montañosas [5]. Si bien muchas personas recurren al uso de un dispositivo móvil para ubicarse en lugares de montaña, no es tan confiable utilizarlo, debido a problemas como falta de señal o batería, además, no está centrado en informar sobre los peligros de la zona o los riesgos inminentes.

Debido a los factores mencionados y la creciente problemática, se ha definido la necesidad de crear un objeto inteligente (un objeto con capacidades de Internet of Things, que es digital, autónomo y con control de recursos disponibles), con el objetivo de orientar a los visitantes de entornos turísticos de montaña. El propósito principal del proyecto es brindar a los visitantes información de ubicación e interés sobre las instalaciones y condiciones de la zona turística de montaña; notificar al usuario sobre condiciones relevantes a la ubicación y seguridad para mantenerse dentro de los límites y alertar sobre situaciones de emergencia, evitando extravíos. Así como incitar el uso incorporando elementos estéticos, de usabilidad y ecológicos acordes a la zona que capten la atención, además, que dé resistencia a condiciones climáticas y el deterioro.

Debido a la situación de la pandemia y la modalidad virtual de investigación, se propone el desarrollo de simulaciones por medios digitales para ver el impacto que tendría el objeto en relación con la problemática presente.

\section{Método}

La metodología empleada para la creación de este proyecto se planteó iniciando desde lo primordial del objeto, como su conceptualización, y con base en lo definido, desarrollar su funcionalidad, seguido de su forma, para luego plantear su manufactura y realizar una simulación de diseño del objeto. A continuación, se detallan las etapas desarrolladas en el proyecto.

\section{Conceptualizando la idea}

En esta etapa se empezó explorando los tipos de personas que han frecuentado los lugares de turismo de montaña, preguntando sus actividades en el lugar, las problemáticas enfrentadas y otras situaciones que hayan generado molestias en sus experiencias, esto por medio de una encuesta inicial. Con base en los resultados de la encuesta, se plantearon ciertas problemáticas y necesidades que las personas tienen en los lugares de turismo de montaña. Además, se investigó en noticias sobre problemas en Costa Rica en cuanto al turismo de montaña y la manera en la que son abordados. 
Con base en la investigación, se definió una lista de necesidades y requerimientos a cubrir por el objeto, entre ellos están: información de la ubicación en el lugar, ubicación clara de instalaciones y servicios, informar/alertar si no se encuentra en ruta correcta, entre otros. Así mismo, se definió un segmento de mercado al cual se dirigiría el producto basado en las personas encuestadas y datos de instituciones como el Instituto Costarricense de Turismo y el Sistema Nacional de Áreas de Conservación, como se ve en la figura 1. Se cuenta con el total anual de visitas en los volcanes y parques nacionales.|

\section{TAMAÑO DE MERCADO}

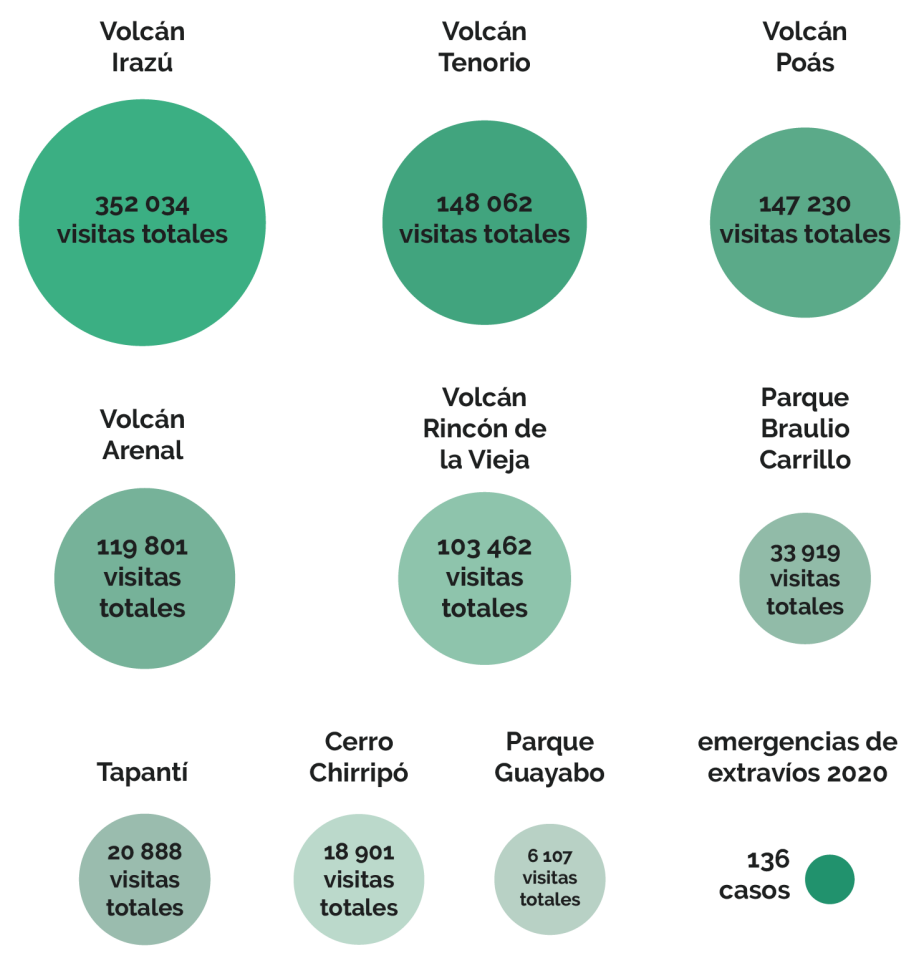

Fig. 1. Cantidad de visitas anuales a los parques y volcanes del país. [2]

Se definieron cuatro personas principales a considerar durante la realización del proyecto, las cuales son: senderistas, padre de familia, turista y extranjero. Con base en estas, se realizó una evaluación de los requerimientos planteados, tomando en consideración usuarios que calzaran en el perfil de las personas planteadas.

Además, se realizó un análisis de referenciales de objetos creados para informar a personas en lugares turísticos, así como objetos centrados en ayudar a personas en situaciones de riesgo en montañas y terrenos complejos. El análisis se da con el fin de identificar la manera de abordar cada proyecto, en temas como su conceptualización, la función a cumplir, sus materiales y formas, así como tecnologías implementadas. 


\section{Definiendo la funcionalidad}

Durante esta fase, se comenzó a definir aspectos y necesidades importantes del producto inteligente desde un punto de vista de la función, tales como sistemas y subsistemas del producto, funciones inteligentes y mecánicas, aspectos perceptuales y emocionales del producto, entre otros.

El desarrollo de la etapa se hizo por medio de un análisis tecnológico, revisando principios de funcionamiento y realizando varios esquemas y diagramas, como se puede observar en la figura 2, para tener claridad sobre cuál es la funcionalidad del producto.

\section{Diagrama sistemas y subsistemas}

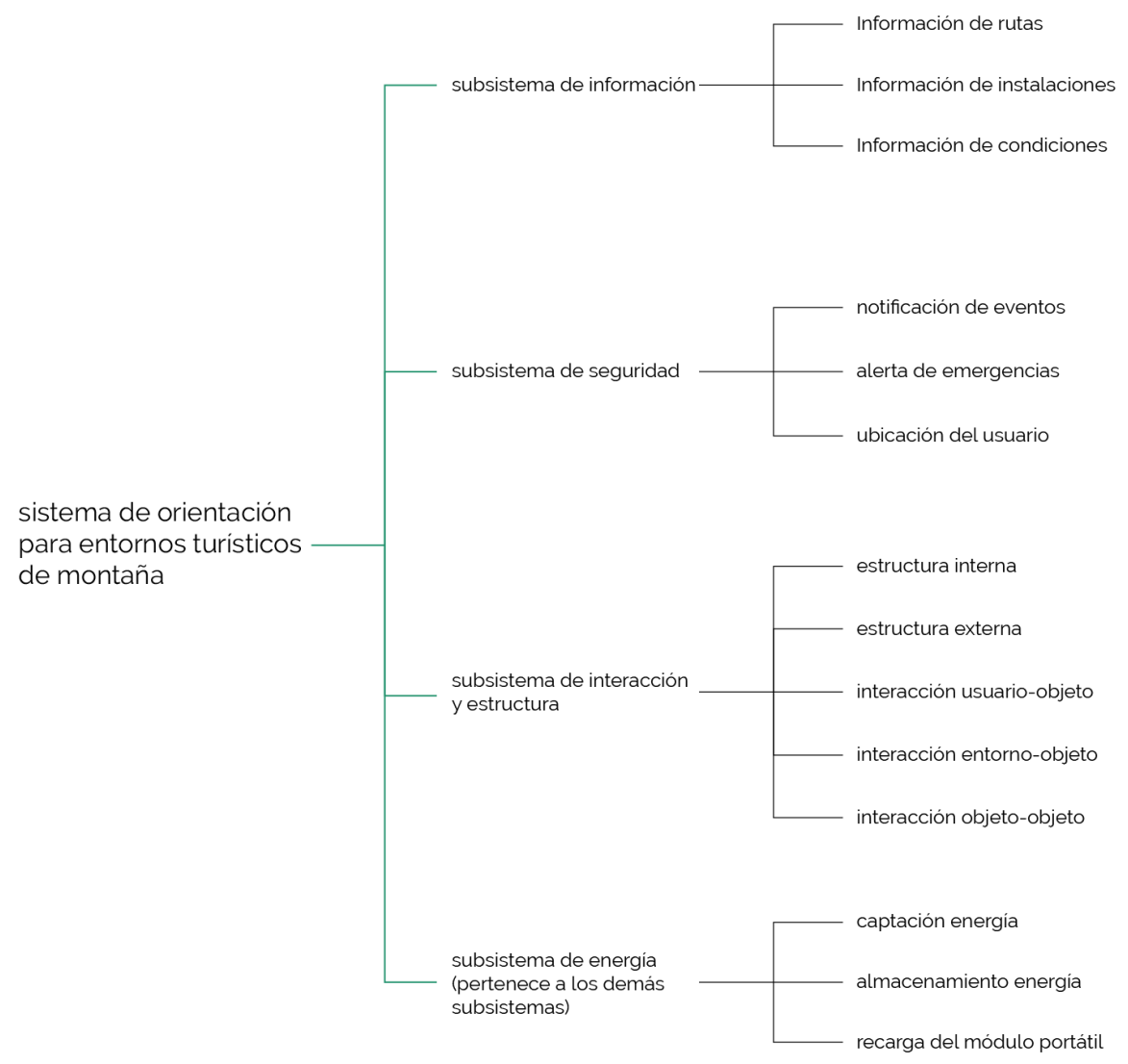

Fig. 2. Diagrama de sistemas y subsistemas.

Una vez definidos estos aspectos, el equipo de diseño logró percibir que, para suplir todas las funciones con las cuales debería contar este producto, fue necesario segmentarlo en dos subsistemas clave, los cuales son: un tótem como subsistema fijo y el collar portátil; además de realizar una encuesta con usuarios de los segmentos de mercado para validar si las funciones definidas estaban bien orientadas a resolver los problemas que tienen y cubrir las necesidades requeridas. 


\section{Definiendo la forma}

Para esta etapa, se definió el concepto de diseño, objetivos, alcances, expectativas y se dio una respuesta a las preguntas: ¿qué es?, ¿para quién?, ¿para qué?, ¿cómo? y ¿dónde?, con el fin de definir lo que se quiere lograr con el producto, orientando su diseño desde este punto de partida.

Continuando en la etapa, se realizó un análisis ergonómico, para definir aspectos formales y cognitivos importantes con los que el producto debería cumplir, como alturas, anchos, simbologías, estímulos requeridos, basados en datos antropométricos de la población latinoamericana [5]. También se analizaron datos de biomecánica para conocer sobre el espacio y posiciones de los elementos interactivos del objeto [6]. Además de un análisis perceptual para definir aspectos de la estética y cómo se necesita que el producto se perciba, esto por medio de referenciales, moodboards y ejes semánticos.

Tomando en cuenta la información recopilada de dichos análisis y del concepto de diseño, se realizaron bocetos de diferentes propuestas, evaluándolos respecto a los requerimientos y necesidades, para seleccionar una propuesta a la cual se aplicaron mejoras. Así mismo, se detalló considerando la perceptibilidad y mejoras encontradas en las demás propuestas y las necesidades a cumplir por medio de bocetos, modelado y renderizado 3D.

\section{Detallado de la manufactura}

Considerando que el curso se impartió en modalidad remota, debido al contexto actual de pandemia, se planteó la producción de forma en la que se detallaran los procesos de manufactura y construcción del producto apoyándose de recursos virtuales como el modelado y renderizado 3D, explicando las generalidades del producto por estos medios.

Se investigó sobre componentes normalizados y materiales disponibles en el mercado, con el fin de hacer una selección correcta tanto de dichos componentes como de los procesos de manufactura idóneos para el producto, además de considerar los posibles costos para así realizar una cotización realista y detallada.

Se planteó un manual de usuario donde se incluyen aspectos de usabilidad del tótem y collar, considerando también la instalación en el lugar y mantenimiento requerido, con el fin de orientar a los diferentes usuarios y personas que se ven involucrados con el producto.

\section{Simulación diseño}

Debido a la falta de una validación física del diseño del producto por la situación de pandemia del COVID-19, se plantearon diferentes simulaciones para obtener una validación por medio del uso de renderizado 3D de alta calidad, así como un video explicativo de las funciones y la interacción con el usuario, con la intención de que cualquier persona pueda entender el producto y sus usos [8]. 


\section{Discusión de resultados}

A partir de la investigación y analizando los atributos sobre seguridad turística necesarios para brindar una buena experiencia, tales como protección de su vida, salud, integridad física, psicológica y económica [3, p. 258], junto a los hallazgos realizados en las diferentes etapas,

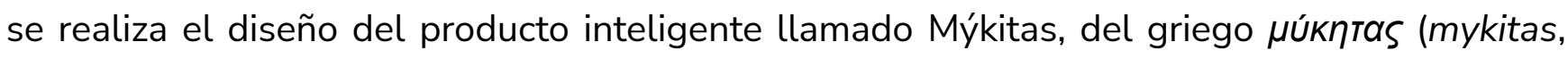
'hongo'), en alusión a su forma similar a un hongo, cuya función es brindar asistencia de emergencia y orientación al usuario mientras realiza senderismo, de manera que brinde una mayor sensación de seguridad al usuario durante su visita.

El propósito principal del sistema, definido en las primeras etapas del proyecto, es brindar a los visitantes información de ubicación e interés acerca de las instalaciones y condiciones de la zona turística de montaña; notificar al usuario sobre condiciones relevantes a la ubicación y seguridad, para mantenerse dentro de los límites y alertar sobre situaciones de emergencia, evitando extravíos; e incitar su uso al incorporar elementos estéticos, de usabilidad y ecológicos acordes a la zona, que capten la atención y brinden resistencia a condiciones climáticas y al deterioro. Se consigue crear el producto inteligente con las características indicadas anteriormente, logrando así proporcionar al usuario seguridad y orientación durante el senderismo, así como brindar una experiencia grata y agradable, con el fin de promover el turismo de montaña.

\section{¿Cómo se resuelve el problema?}

El diseño del producto Mýkitas resuelve una problemática existente en el turismo de montaña, de manera que, al constituirse de dos elementos: uno estático y otro portátil, facilita el monitoreo de cada persona y que sean más fáciles de localizar para el personal del parque; en caso de una emergencia, evita la necesidad de llevar equipo de más, ya que se cuenta con la ubicación exacta.

El collar localizador Mýkitas acompaña al usuario en todo momento y le brinda la oportunidad de una mayor independencia en su camino, además de la posibilidad de pedir ayuda en cualquier momento que lo requiera.

Los productos se mantienen interconectados por medio de radiofrecuencia, esto permite que haya comunicación entre ellos, independientemente de la lejanía a la que se encuentre la persona y, si la persona llega a estar en una ruta incorrecta o alejada de los rangos permitidos, el collar dará diferentes alertas al usuario de manera inmediata para informar sobre el riesgo. Se detalla la arquitectura del producto con el uso de renders en explosos tanto del tótem como del collar, mostrando detalles de unión y ensamble, materiales, sistemas y subsistemas que contiene (figura $3,4,5$ y 6 ). 


\section{Tótem: partes externas}

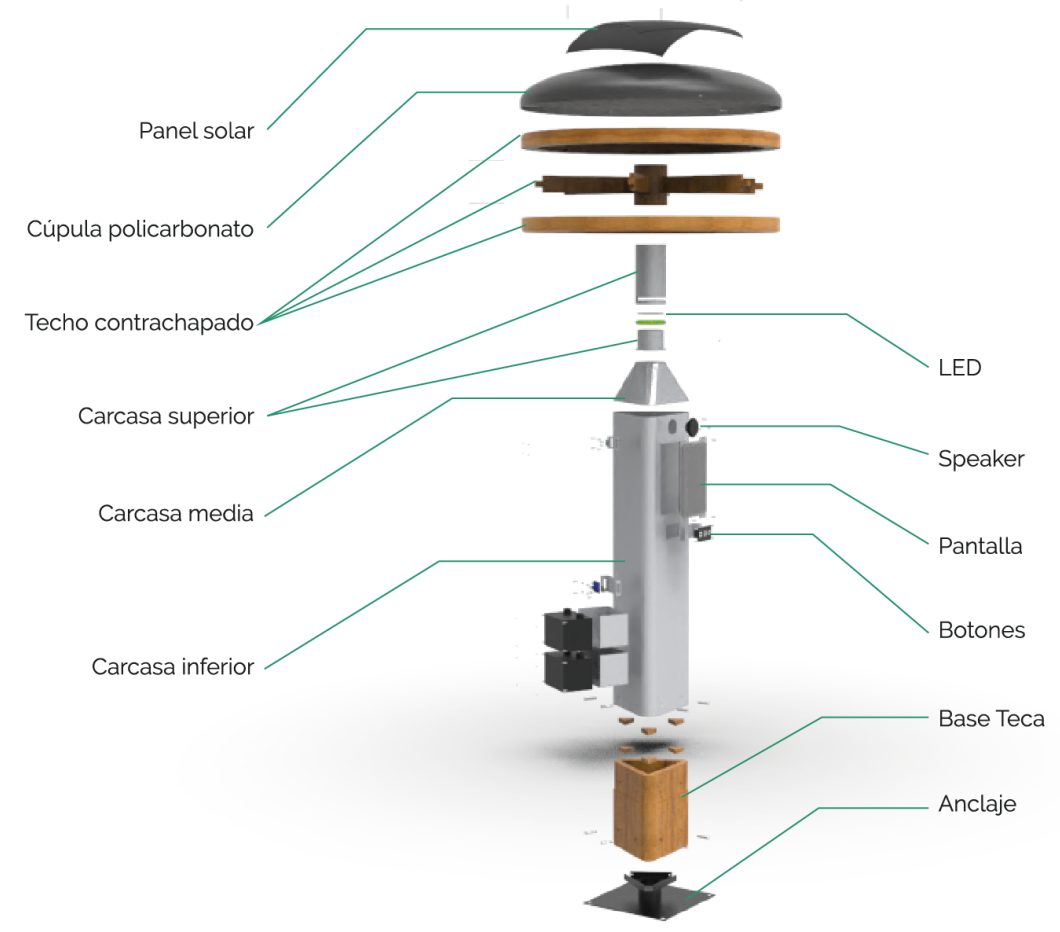

Fig. 3. Exploso arquitectura del Tótem.

\section{Localizador: partes}

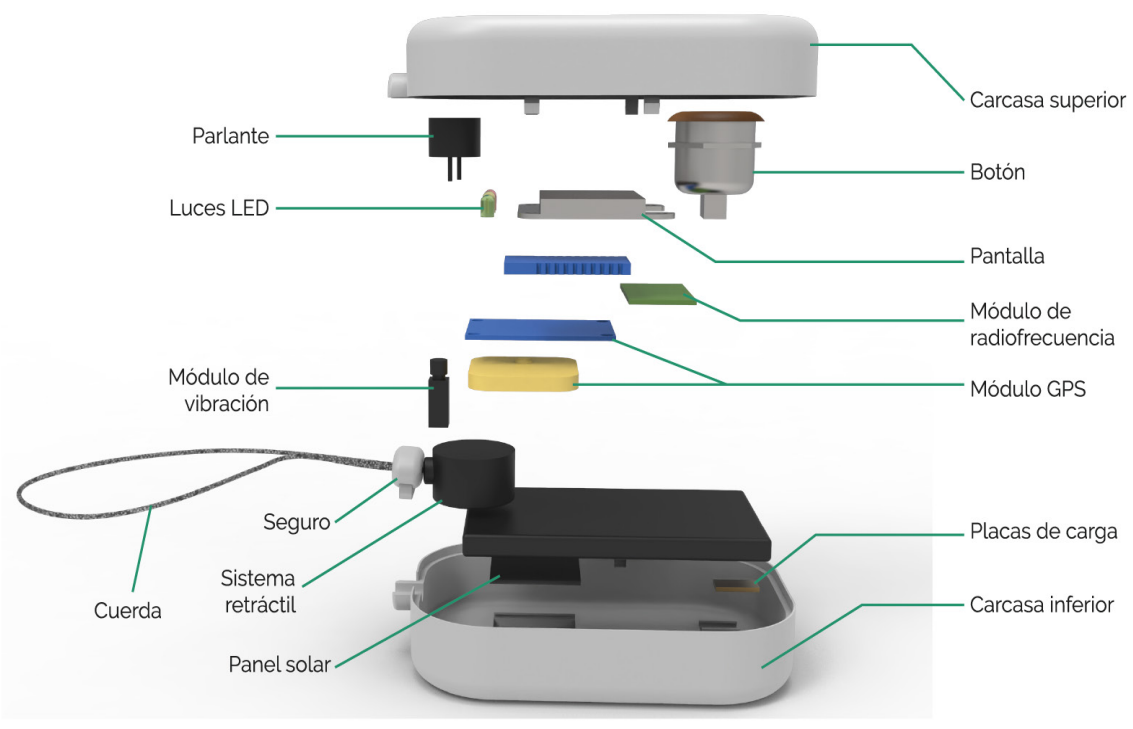

Fig. 4. Exploso arquitectura del localizador. 


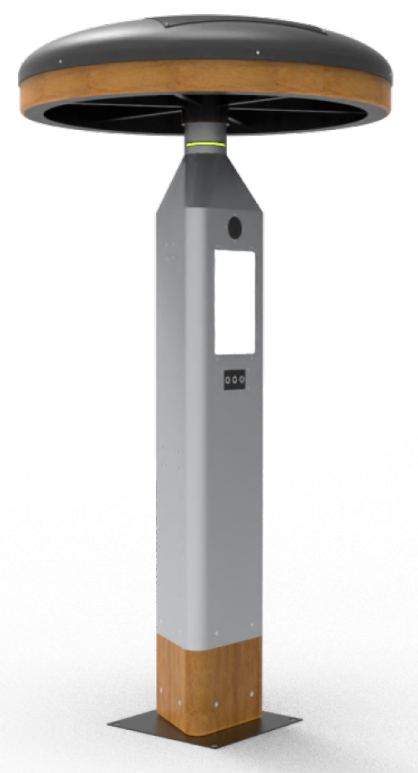

Fig. 5. Render del tótem.

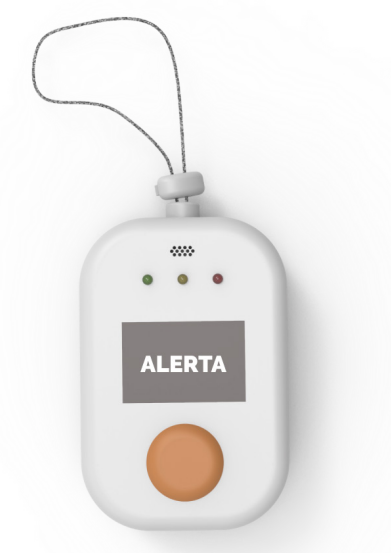

Fig. 6. Render del localizador.

\section{Hallazgos técnicos/manufactura}

Para la fabricación de las partes, se sugieren materiales que resistan el ambiente complejo de montaña, el cual implica grandes cantidades de lluvia, humedad y polvo. El techo del tótem consta de madera contrachapada, ya que presenta mayor resistencia para soportar dicha estructura.

La base del tótem se construye a partir de tablas de madera de teca unidas y cuñas del mismo material en la parte interior para brindar mayor estabilidad y soporte. Para reforzar toda la estructura del tótem, se adiciona una capa de acero entre el suelo y el objeto inteligente. 


\section{Uso del tótem y collar localizador Mýkitas}

En la figura 7, se puede observar el uso y la interacción, además de las funciones inteligentes que posee el producto. Se agrega un video explicativo [8] donde se plantean las interacciones del producto con los usuarios.

En el video se observa cómo se brindará un localizador Mýkitas al usuario al ingresar a un parque, para que, por medio de este, pueda enlazarse con los tótems y recibir información. Si el usuario se sale de las rutas seguras en los senderos, el collar brindará señales de alerta con luz, sonido y vibraciones, y en la aplicación podrá encontrar una ruta adecuada para volver. En caso de ocurrir un accidente o emergencia, el collar tiene un botón para llamar a las autoridades correspondientes y ser atendido.

Uso e interacción del producto:

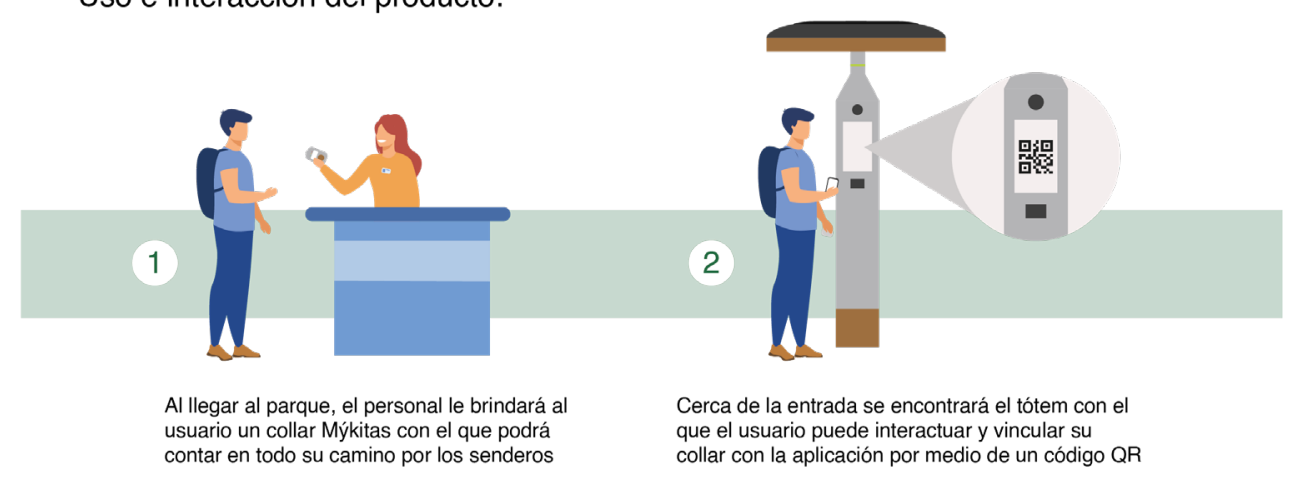

En caso de extravío:

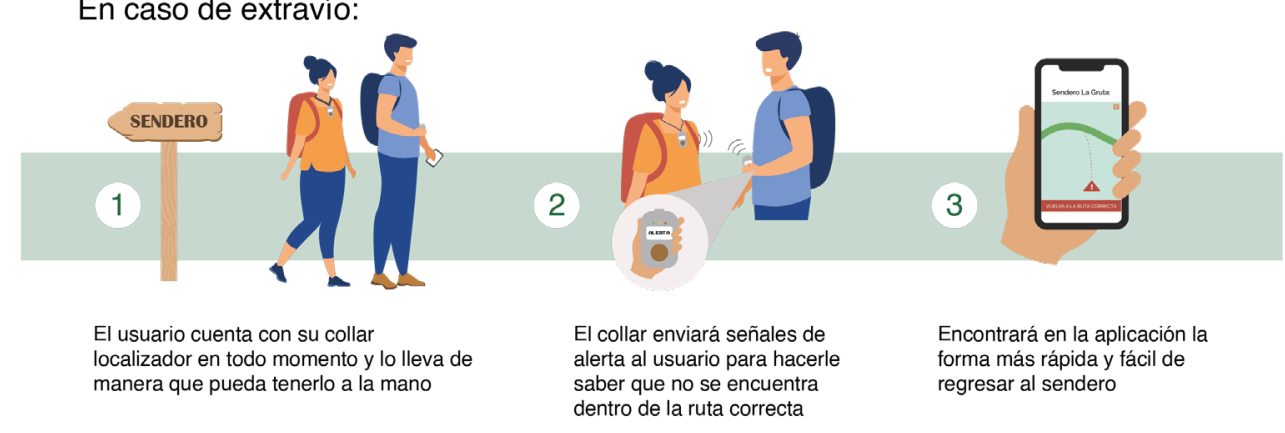

En caso de emergencia:

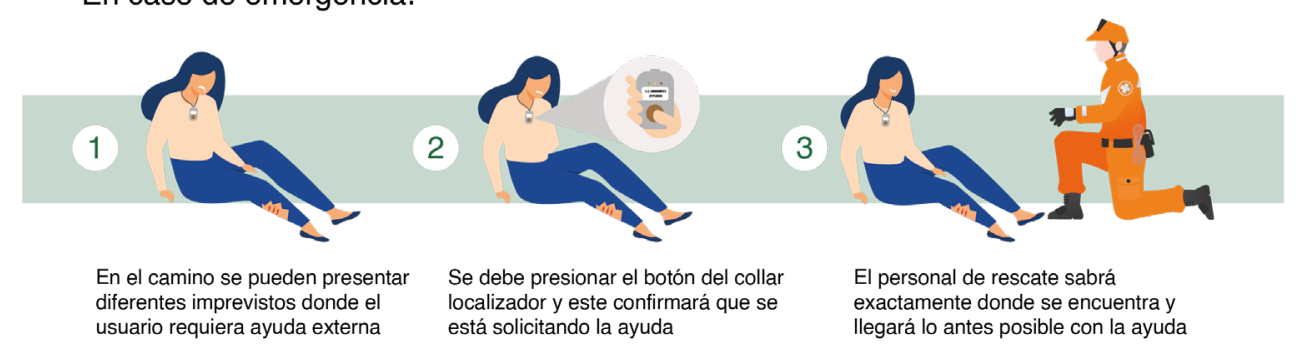

Fig. 7. Uso e interacción del producto inteligente. 


\section{Conclusiones}

Contar con el producto inteligente Mýkitas, al realizar senderismo en los principales parques del país, representará una gran ayuda para los visitantes y el personal de los parques. Las personas logran sentirse más seguras en su camino, ya que pueden acceder a ayuda en cualquier momento. También se logra una mayor independencia en los usuarios, gracias a que, al contar con la aplicación en sus teléfonos, pueden tener el mapa de los senderos en todo momento y estar seguros de por donde caminan. Además, se mejora la experiencia del usuario mientras realiza senderismo, ya que puede saber información como datos interesantes del lugar y condiciones de este. Al momento de una emergencia, la utilización de este producto inteligente permite que se pueda brindar una respuesta más rápida para las personas afectadas.

Otros beneficios que se adquieren al contar con este producto son los económicos, ya que permite conocer el lugar exacto de la persona afectada en una emergencia y se disminuye el personal y equipo de rescate. Al encontrarse ubicado dentro de las instalaciones de los parques, el producto brinda un factor de estética y tecnología sin perder la naturalidad. A su vez, el turismo costarricense de montaña se beneficia mejorando su imagen y atrayendo a cada vez más público tanto nacional como extranjero.

\section{Referencias}

[1] Informe anual Estadísticas SEMEC 2017, Sistema Nacional de Áreas de Conservación, [En línea]. Disponible: http://www.sinac.go.cr/ES/transprncia/Informe\%20SEMEC/ Informe\%20SEMEC\%202017.pdf

[2] Visita a las Áreas Silvestres Protegidas 2019 (SINAC), Instituto Costarricense de Turismo, [En línea]. Disponible: https://www.ict.go.cr/es/documentos-institucionales/ estad\%C3\%ADsticas/cifras-tur\%C3\%ADsticas/visita-a-las-\%C3\%A1reas-silvestresprotegidas-sinac/1397-2017-2/file.html

[3] R. Martínez y O. Trejoluna. "La percepción de seguridad de los turistas en un sitio de turismo religioso." en International Journal of Scientific Management and Tourism, 2017. pp. 255-273, [En línea]. Disponible: https://dialnet.unirioja.es/descarga/articulo/6182550. pdf.

[4] La Nación. Cruz Roja reporta incremento de rescates en montañas desde que comenzaron restricciones por pandemia Ago. 2020. [En línea]. Disponible: https:// www.nacion.com/sucesos/seguridad/cruz-roja-reporta-incremento-de-rescatesen/5XM36AIMGNETRGNGXRE6MV7VOI/story/

[5] La Nación. ¿De paseo a la montaña? Conserve carga en el celular porque podría salvarlo en caso de extravío. Ene, 2019. [En línea]. Disponible: https://www. nacion.com/sucesos/seguridad/de-paseo-a-la-montana-conserve-carga-enel/26P6HLY42JEZBBQAMN7ALFTALU/story/ 


\section{revistaIDI+}

[6] A.C. Rosalio, "Dimensiones antropométricas de la poblaciónlatinoamericana : México, Cuba, Colombia,Chile". [En línea]. Disponible:

https://repository.usta.edu.co/bitstream/handle/11634/14486/2018sergioboh\%C3\%B3rquez4. pdf? sequence $=6 \&$ is Allowed $=y$

[7] Araya, L; "Ángulos de movimiento, laboratorios biomecamica”.2018. [En línea]. Disponible: Tecdigital.

[8] A. Piedra, B. Quirós, A. Rojas. "MÝKITAS: Diseño de producto inteligente para el turismo de montaña.". Jun. 2021. [En línea]. Disponible: https://www.youtube.com/ watch?v=R4LO0GGTMAw 